
\title{
BReserch S Suare \\ Shifts in Help-seeking Patterns During COVID-19: Should Social Distancing be Rebranded?
}

\author{
Alvin Junus \\ University of Hong Kong \\ Ching Kwan \\ University of Hong Kong \\ Clifford Wong \\ University of Hong Kong \\ Zhansheng Chen \\ University of Hong Kong \\ Paul Yip ( $\nabla$ sfpyip@hku.hk ) \\ University of Hong Kong
}

\section{Research Article}

Keywords: help-seeking pattern, social support, youths, stressors, perceived distress, social distancing

Posted Date: July 7th, 2021

DOl: https://doi.org/10.21203/rs.3.rs-668704/v1

License: (c) (i) This work is licensed under a Creative Commons Attribution 4.0 International License. Read Full License 


\title{
Shifts in Help-seeking Patterns During COVID-19: Should Social Distancing be Rebranded?
}

\author{
Alvin Junus ${ }^{1}$, Ching Kwan ${ }^{2}$, Clifford Wong ${ }^{2}$, Zhansheng Chen ${ }^{3}$, and Paul Siu Fai Yip ${ }^{1,2,{ }^{*}}$ \\ ${ }^{1}$ Department of Social Work and Social Administration, The University of Hong Kong \\ ${ }^{2}$ The Hong Kong Jockey Club Centre for Suicide Research and Prevention, The University of \\ Hong Kong \\ ${ }^{3}$ Department of Psychology, The University of Hong Kong \\ * corresponding author: sfpyip@hku.hk
}

\begin{abstract}
Much focus has been placed on mental health symptoms brought forth by the COVID-19 pandemic, yet limited discourse \& evidence have evaluated how the closure of multiple venues under social distancing measures impacted people's patterns of help-seeking, which had traditionally been the very coping mechanism that buffered individuals from the consequences of those studied symptoms.

Using a two-wave (June-July 2019 and June-September 2020) panel data on youths aged 11-35 years old, the present study shows that under social distancing, a significant proportion of individuals who used to rely on their strong ties for support no longer sought help even after controlling for stress level changes \& sociodemographic factors, and only those who were facing heavier distress ended up seeking their strong ties for support.

By simply closing off social contexts that had traditionally facilitated social support provision among strong ties and not providing people with alternative contexts, current social distancing measures appeared to have effectively increased the difficulty for many to receive social support, thereby leaving them vulnerable to mental health impacts of the pandemic. To prevent the current pandemic from turning into a mental health pandemic, the mantra "social distancing" should be revised to encourage society to remain socially close even while physically distant.
\end{abstract}

Keywords — help-seeking pattern; social support; youths; stressors; perceived distress; social distancing 


\section{Introduction}

One's social network is inextricably linked to their mental health, chiefly due to the intrinsic social support and social integration that it provides them with $[1,2,3,4]$. While numerous studies over the years have reliably established that these two factors protect individuals from suicidal behaviours and other adverse mental health outcomes $[2,5]$, it is also widely understood that the individual themself needs to activate their social network ties in order to derive these forms of support $[2,6,7]$, i.e., to actively seek help from their relationships.

From a social capital perspective, this can be explained with the concept of bonding social capital [8]: People need to invest their time engaging in shared activities together to cultivate their social bonds, facilitating resources being shared and foster feelings of relatedness, mattering, and a sense of belonging, and ultimately strengthening their social ties over time [9]. In turn, one's bonding social capital allows them to mobilise resources embedded in their strong ties when needed (e.g., when they face stressors) in the form of resource-sharing, provision of social and emotional support, etc., thus influencing their trajectory from an episode of distress to major psychiatric conditions $[6,10,11,12,13]$. In this regard, shopping venues, bars, restaurants, cafes, cinemas, parks, places of worship, and even schools serve as contexts not only for individuals to cultivate their bonding social capital but also for affirmative social support to flow through strong ties [14].

During this COVID-19 pandemic, many societies have strongly enforced lockdowns and social distancing measures, which required people to maintain physical distance and limit interactions with others. With many bonding social capital contexts closed down as part of social distancing measures, people's means of help-seeking were restricted. Coupled with these restrictions, the emphasis placed on the term "social distancing", as mental health scientists argued, would spur people to internalise this policy, instilling a sense of a break in social connections [15] and invoking feelings of loneliness \& social isolation. Ultimately, people could be left more vulnerable to adverse mental health effects caused by the pandemic $[15,16,17,18]$.

Within the past year, there had thus been many studies focusing on the incidence of symptoms such as depression, distress, loneliness, and anxiety arising due to these measures [19, 20, 21]. Yet, they had largely left out a subtle yet important aspect - people's help-seeking behaviours in response to adverse life events - which had traditionally been the very mechanism that mitigated the psychological impact of the stated symptoms on their mental health. An understanding of the shifts in people's help-seeking patterns is thus urgently needed to prevent the current pandemic from bringing forth a mental health pandemic. Therefore, in this paper we investigated the question: How had social distancing measures altered people's tendencies to seek help when facing stressors in their lives?

Since social distancing measures restricted people's access to bonding social capital contexts, we hypothesised that: (H1) Social distancing measures lowered people's tendencies to seek help when facing stressors in their lives. Stress intensity and the tendency to seek help, however, are known to be associated with each other [22, 23]; the higher an individual's perceived stress is, the more likely will they seek help. Lockdown and social distancing measures had been expected to exert an unequal impact on different sections of society $[24,25,26]$. This inequality might then manifest among these subgroups as relatively lower levels of distress, and subsequently a lower tendency for them to seek help compared to other subgroups.

Indeed, while people's mental health after the pandemic had fared worse compared to pre-pandemic times $[21,27,28]$, studies confirmed that stress levels of females, children \& adolescents, persons with lower socioeconomic status and pre-existing mental health conditions, as well as minority groups around the world had been disproportionately affected during the pandemic $[28,29,30,31,32,33,34,35]$. Yet, the population in countries such as Germany and China have also shown resilience against lockdown and social distancing measures, showing no clinically significant changes in anxiety, depression, and distress $[27,36,37]$. These studies suggest that stress levels in individuals as well as sociodemographic factors ought to be ruled out before we could deduce the effects of social distancing on people's help-seeking tendency.

Informed by the current literature, we refined our hypothesis: (H2) Controlling for stress levels and sociodemographic factors, social distancing measures still lowered people's tendencies to seek help when facing stressors in their lives. 


\section{Results}

\section{Descriptive statistics and trends in the study population}

Results in this study were drawn from analyzing a two-wave (June-July 2019 and June-September 2020) panel data that assessed youths aged 11-35 years old on their sociodemographics, distress, and help-seeking behaviors. A total of 453 individuals met the inclusion criteria for our study. Table 1 shows descriptive statistics of the study population. The $70.2 \%$ share of females in this sample is appreciably higher than the $51 \%$ share in the general population as documented in the 2016 population by-census [38]. Given the recruitment channels of the surveys, however, this ratio might signify that more females were in touch with community pastoral services, which in turn reflects the prevailing notion that females are more inclined to seek help for mental health problems $[22,39,40]$. The study population's risk behaviour profile also showed elevated mental health risk levels, which may again be attributed to the survey's recruitment channels. Study samples' mean age and standard deviation were 25.66 and 5.05 respectively.

$\begin{array}{llllll} & \mathbf{n} & \mathbf{\%} & & \mathbf{n} & \mathbf{\%} \\ \text { Age group } & & & \text { Currently living with } & & \\ 15-19 & 25 & 5.5 & \text { Alone } & 21 & 4.7 \\ 20-24 & 206 & 45.5 & \text { Family members } & 412 & 92.4 \\ 25-29 & 101 & 22.3 & \text { Friends } & 13 & 2.9 \\ 30+ & 121 & 26.8 & \text { Others } & 7 & 1.6 \\ \text { Gender } & & & \text { Family structure } & & \\ \text { Female } & 318 & 70.2 & \text { Two-parent family } & 377 & 83.4 \\ \text { Male } & 135 & 29.8 & \text { Divorced parents } & 47 & 10.4 \\ \text { Education } & & & \text { Step-family } & 3 & 0.7 \\ \text { Post-secondary or abover } & 403 & 89.0 & \text { One parent passed away } & 24 & 5.3 \\ \text { Secondary school } & 47 & 10.4 & \text { Both parents passed away } & 1 & 0.2 \\ \text { Primary school } & 0 & / & \text { Missing } & 1 & 0.2 \\ \text { Refuse to answer } & 3 & 0.7 & \text { Suicidal risk behaviours } & & \\ \text { Occupation status } & & & \text { Considered suicide } & 70 & 15.5 \\ \text { Full-time } & 258 & 57.0 & \text { Attempted suicide } & 11 & 2.4 \\ \text { Part-time } & 64 & 14.1 & \text { Intentional self-harm } & 39 & 8.6 \\ \text { Unemployed } & 131 & 28.9 & & & \end{array}$

Table 1: Descriptive statistics of the study population as of the $3^{\text {rd }}$ survey wave. Percentage points are rounded to 1 decimal place.

Between-wave changes in distress, social withdrawal screenings, and help-seeking tendencies of the study population are listed on Table 2. Even though the number of individuals who fulfilled the criteria for social withdrawal increased from 54 to 129 , this does not necessarily imply that they became Hikikomoris in the past year; rather, a more plausible explanation might be that the imposed social distancing measures had compelled them to spend most of their days confined at home and to avoid social situations \& social contact, which incidentally fulfilled diagnosis criteria for Hikikomori but also showed that the study population had experienced social distancing.

More importantly, however, the number of individuals who sought help in response to distress fell considerably, from 299 before the pandemic to 168 afterwards, even though their average stress levels in both waves largely stayed the same $(t=0.08, p=0.93)$. In particular, activation of family, friends, partner, and religious services channels was significantly lower compared to the previous year's, while the other six channels did not show a statistically significant drop. (It is, however, noteworthy that treatments for clinical and more serious mental conditions, e.g., psychiatric appointments, hotline counseling support, social services, etc., still continued even amidst social distancing measures, as evidenced by their consistent activation of formal support channels in both waves.)

LCA of survey participants' help-seeking behaviours would tell us whether these decreases in help-seeking tendencies were contributed by the whole study population or could rather be attributed to just specific individuals. 


\begin{tabular}{lllll} 
& $\mathbf{2 0 1 9}$ & $\mathbf{2 0 2 0}$ & Statistical tests & Effect size \\
\hline Stress levels [Mean (s.d.)] & $2.59(0.76)$ & $2.59(0.79)$ & $t(902.67)=0.08, p=0.93$ & $d=0.01[-0.18,0.18]$ \\
CHQ-12 score [Mean (s.d.) & $21.71(6.30)$ & $21.73(6.09)$ & $t(902.98)=-0.04, p=0.97$ & $d=-0.00[-0.18,0.19]$ \\
Social withdrawal & $54(11.9 \%)$ & $129(28.5 \%)$ & $\chi^{2}=37.50, p<0.001^{* * *}$ & $O R=2.94[2.05,4.26]$ \\
\# of individuals who sought help & $299(66.0 \%)$ & $168(37.1 \%)$ & $\chi^{2}=74.69, p<0.001^{* * *}$ & $O R=0.33[0.25,0.44]$ \\
from family & $112(24.7 \%)$ & $67(14.8 \%)$ & $\chi^{2}=13.48, p<0.001^{* * *}$ & $O R=0.53[0.37,0.75]$ \\
from friends & $231(51.0 \%)$ & $133(29.4 \%)$ & $\chi^{2}=43.21, p<0.001^{* * *}$ & $O R=0.40[0.30,0.53]$ \\
from partner & $118(26.0 \%)$ & $58(12.8 \%)$ & $\chi^{2}=24.55, p<0.001^{* * *}$ & $O R=0.42[0.29,0.60]$ \\
from teacher / tutor & $26(5.7 \%)$ & $16(3.5 \%)$ & $\chi^{2}=2.02, p=0.16$ & $O R=0.60[0.30,1.18]$ \\
from hotline support & $11(2.4 \%)$ & $4(0.9 \%)$ & $\chi^{2}=2.44, p=0.12$ & $O R=0.36[0.08,1.22]$ \\
from medical professionals & $20(4.4 \%)$ & $14(3.1 \%)$ & $\chi^{2}=0.76, p=0.38$ & $O R=0.69[0.32,1.46]$ \\
from social workers & $39(8.7 \%)$ & $27(6.0 \%)$ & $\chi^{2}=1.98, p=0.16$ & $O R=0.67[0.39,1.15]$ \\
from religious services & $22(4.9 \%)$ & $5(1.1 \%)$ & $\chi^{2}=9.77, p=0.002^{* *}$ & $O R=0.22[0.06,0.60]$ \\
from online friends & $25(5.5 \%)$ & $16(3.5 \%)$ & $\chi^{2}=1.64, p=0.20$ & $O R=0.63[0.31,1.24]$ \\
from online social services & $9(2.0 \%)$ & $9(2.0 \%)$ & $\chi^{2}=0.00, p=1.0$ & $O R=1[0.35,2.87]$
\end{tabular}

Table 2: Stress levels, social withdrawal screenings, and activated support channels in the $2^{\text {nd }} \& 3^{\text {rd }}$ survey waves $(n=453)$. Rows are in a $[n(\%$ of study population $)]$ format unless indicated otherwise. Numbers are rounded to 2 decimal places and percentage points are rounded to 1 decimal place. $\quad *: p<0.05, * *: p<0.01, * * *: p<0.001$.

\section{Shifts in help-seeking patterns}

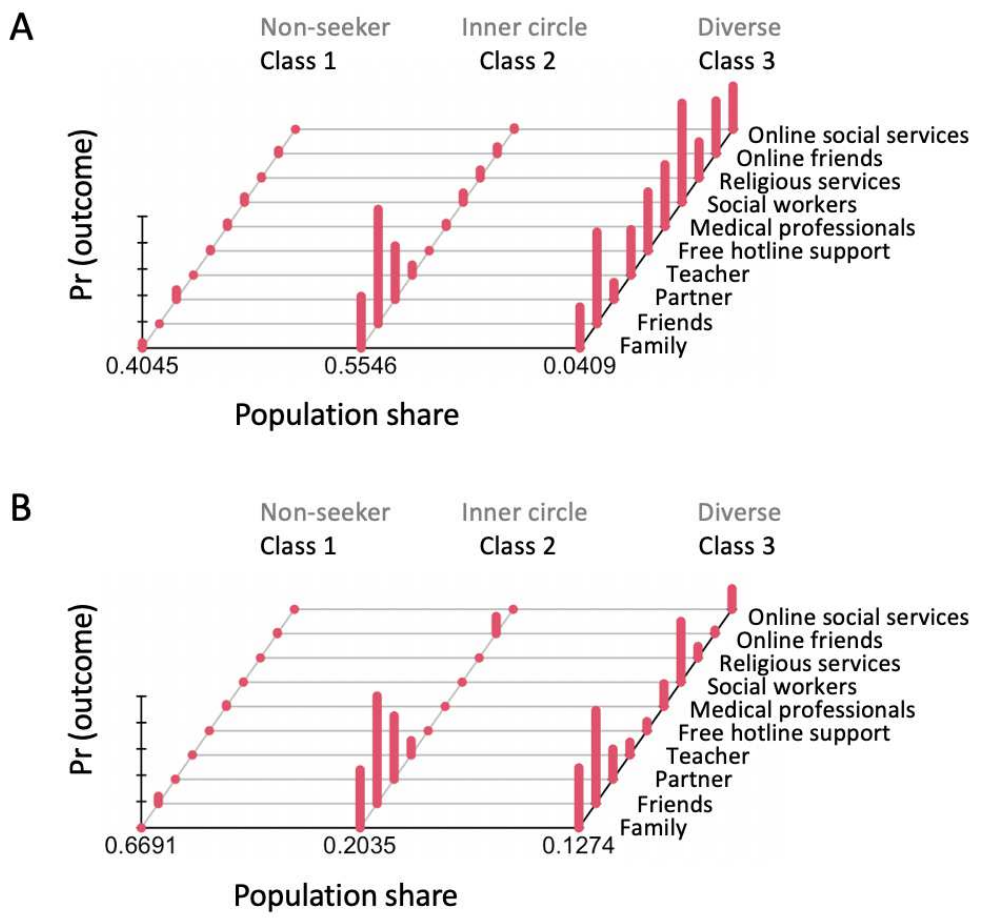

Figure 1: Optimal model (based on model-fit parameters \& interpretability) for patterns of help-seeking behaviours within the study population in (A) 2019 and (B) 2020. Each class' estimated proportion of the total study population is shown on the horizontal axis, while the vertical axis denotes the estimated probability of an individual seeking support from a corresponding channel.

Model-fit parameters and the interpretability of each class pointed to an optimum of 3 latent classes for both survey waves. Figure 1 illustrates the estimated size and composition of latent classes of help-seeking behaviours in 2019 \& 2020. The same patterns were observed in both waves. Accordingly, individuals in the study population could then be classified into three types based on their help-seeking patterns: the first class of individuals, whom we refer to as Non-seekers, sought minimal help in relation to distress; the second class, Inner circle seekers, sought help from their inner circle sources - family, friends, and partner - for their mental health problems; and the third class, Diverse seekers, reached out to their inner circle as well as formal sources of help for their mental health problems. 
Among the three patterns, Non-seekers had the lowest stress level (mean=2.38, s.d. $=0.76$ ), which reflects the intuition and established understanding that individuals are less likely to seek help if they are only experiencing light psychological distress. In comparison, the Diverse seekers had the highest stress level (mean=3.28, s.d. $=0.77)$ and the Diverse seekers in-between the two (mean=2.85, s.d. $=0.68)$. This agrees with prior understanding that youths who sought formal help showed worse mental health outcomes than those who only sought informal help, e.g., family and friends [41]. Noteworthily, Non-seekers and Diverse seekers showed no significant changes in their stress levels across the two waves but Inner circle seekers in the $3^{\text {rd }}$ wave showed elevated stress levels (summarised in Supplementary Table S2).

Consistent patterns in both waves notwithstanding, Figure 1 shows that the number of Non-seekers ballooned from 0.40 of the study population before the pandemic to almost 0.67 afterwards whereas the number of Inner circle seekers conversely shrunk from around 0.55 to just over 0.20 of the study population. Meanwhile, the change in the share of Diverse seekers was smaller, increasing slightly from 0.04 before the pandemic to around 0.13 afterwards.

More specifically, as the latent class transition matrix in Eq. 1 shows, out of 231 youths who were classified as Inner circle seekers in 2019, 130 shifted to be Non-seekers in 2020. Even though there were others pattern shifts, the $I \rightarrow N$ (Inner circle seekers to Non-seekers) transition was much more prevalent than any other class transition, as the other five possible transitions combined only numbered 84 individuals. This indicates that decreases of activated family, friends, and partner channels for support that was observed in Table 2 are all largely driven by the same individuals who underwent this $I \rightarrow N$ transition. Therefore, H1 is confirmed for those individuals who primarily sought support from their inner circle, i.e., those who primarily faced moderate levels of distress.

$$
S=\left[\begin{array}{ccc}
S_{N \rightarrow N} & S_{N \rightarrow I} & S_{N \rightarrow D} \\
S_{I \rightarrow N} & S_{I \rightarrow I} & S_{I \rightarrow D} \\
S_{D \rightarrow N} & S_{D \rightarrow I} & S_{D \rightarrow D}
\end{array}\right]=\left[\begin{array}{ccc}
155 & 42 & 9 \\
130 & 77 & 24 \\
7 & 2 & 7
\end{array}\right]
$$

\section{Why had so many Inner circle seekers become Non-seekers in 2020?}

Based on the latent class transition matrix, we centred on understanding reasons behind the high prevalence of the $I \rightarrow N$ transition. Individuals with an $I \rightarrow N$ transition had stable mental health, as between-wave difference in their average stress levels was $-0.16(t(256.82)=-1.90 ; p=0.06 ; d=0.24)$. We then compared individuals who underwent a $I \rightarrow N$ transition to those corresponding to other sizeable pattern changes (see Supplementary Table S3).

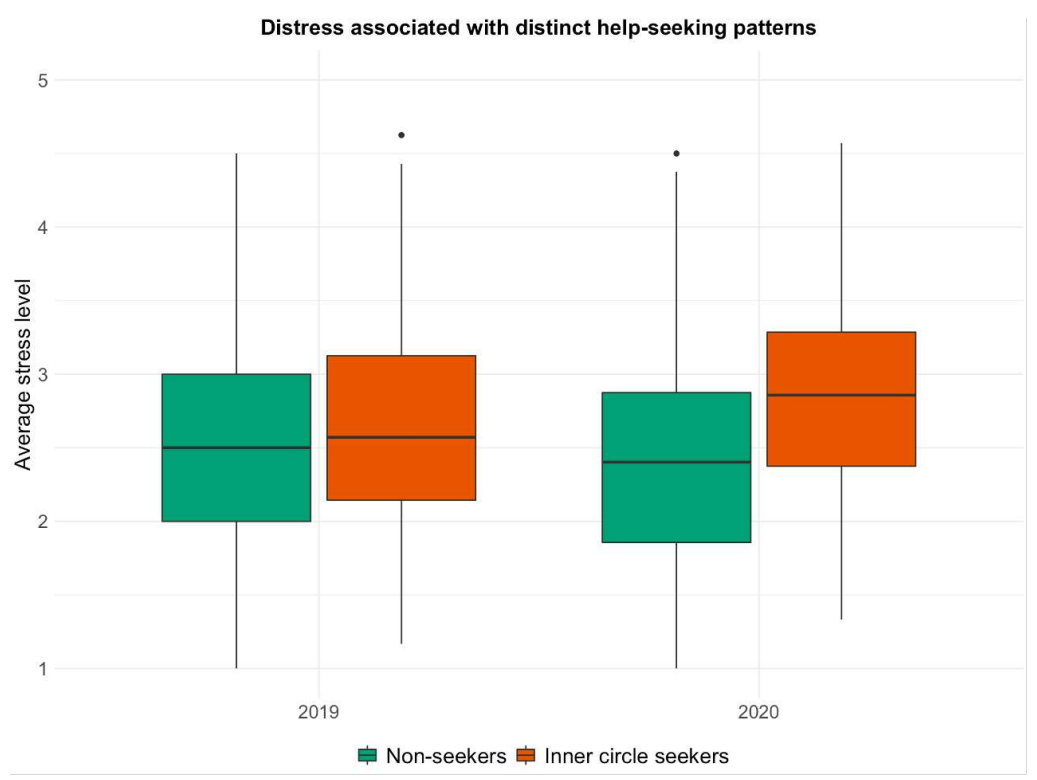

Figure 2: Average stress levels of Non-seekers and Inner circle seekers before and during the COVID-19 pandemic.

Table 3 summarises a multiple logistic regression comparing individuals with $I \rightarrow N$ and $I \rightarrow I$ transitions (see 


\begin{tabular}{|c|c|c|}
\hline Predictors & OR & $95 \%$ CI \\
\hline (Intercept) & 1.08 & {$[0.27,4.60]$} \\
\hline Increased stress level & 0.67 & {$[0.40,1.09]$} \\
\hline \multicolumn{3}{|l|}{ Student status } \\
\hline Full-time student & (ref.) & (ref.) \\
\hline Part-time student & 0.44 & {$[0.10,1.93]$} \\
\hline Non-student & 1.14 & {$[0.45,2.91]$} \\
\hline \multicolumn{3}{|l|}{ Occupation status } \\
\hline Unemployed & (ref.) & (ref.) \\
\hline Full-time job & 0.54 & {$[0.22,1.28]$} \\
\hline Part-time job & 1.14 & {$[0.38,3.59]$} \\
\hline \multicolumn{3}{|l|}{ Age group } \\
\hline $15-19$ & (ref.) & (ref.) \\
\hline $20-24$ & 1.67 & {$[0.23,13.38]$} \\
\hline $25-29$ & 1.27 & {$[0.14,12.03]$} \\
\hline \multicolumn{3}{|l|}{ Gender } \\
\hline Female & (ref.) & (ref.) \\
\hline Male & 1.76 & {$[0.86,3.78]$} \\
\hline \multicolumn{3}{|l|}{ Education level } \\
\hline Secondary & (ref.) & (ref.) \\
\hline Post-secondary or above & 1.60 & {$[0.29,7.49]$} \\
\hline \multicolumn{3}{|l|}{ Currently living with } \\
\hline Family members & (ref.) & (ref.) \\
\hline Alone & 0.99 & {$[0.26,4.20]$} \\
\hline Friends & 0.56 & {$[0.13,2.33]$} \\
\hline \multicolumn{3}{|l|}{ Family structure } \\
\hline Two-parent family & (ref.) & (ref.) \\
\hline Divorced parents & 0.46 & {$[0.16,1.28]$} \\
\hline One parent passed away & 7.51 & {$[1.25,147.92]$} \\
\hline
\end{tabular}

Table 3: Odds ratios and 95\% confidence intervals for a multiple logistic regression $(n=207)$ where the dependent variable denotes that an individual underwent an $I \rightarrow N$ transition. Numbers are rounded to 2 decimal places. *: $p<0.05,{ }^{* *}: p<0.01,{ }^{* * *}: p<0.001$.

Supplementary Table S4 shows additional comparisons, which reach the same conclusion). Here, the dependent variable denotes that an individual underwent the $I \rightarrow N$ transition and the predictors are between-wave stress level difference \& sociodemographic factors. Regression results further show that the $I \rightarrow N$ transition was neither associated with stress level changes nor with any sociodemographic predictor. Accordingly, we can therefore conclude that social distancing measures had indeed caused individuals who had previously been seeking support from their partner, family, and friends ties in dealing with stressors to no longer do so during the pandemic, thus confirming $\mathrm{H} 2$ for these individuals.

This finding is in line with restrictions imposed due to social distancing measures in Hong Kong: While the availability of formal mental health support, e.g., mental health professionals, hotline services, etc., was unaffected, settings such as bars, dining venues, etc., in which casual and/or intimate social support activation \& provision took place among individuals" "inner circle" / strong ties were no longer accessible. Therefore, the implementation of social distancing can be regarded as equivalent to introducing a barrier for individuals to seek help from their family, friends, and partner, which caused them to no longer seek support from these sources unless they faced heavy distress or their mental health deteriorated. This then results in the prevalent $I \rightarrow N$ transition regardless of individuals' stress level difference \& sociodemographic factors and leads to an elevated average stress level among Inner circle seekers in the $3^{\text {rd }}$ wave (summarised in Supplementary Table S2 and illustrated in Figure 2). Viewed from this perspective then, social distancing measures essentially reduced people's help-seeking tendencies by increasing the threshold (i.e., minimum stress level required) for individuals who relied on their family, friends, and partner for support to start seeking help from these sources. 


\section{Discussion}

Early into the pandemic, mental health scientists had advocated for an emphasis of the term "physical distancing" rather than "social distancing", arguing that the latter could be internalised by people, which would subsequently instill a sense of a break in social connections [15] and invoke feelings of loneliness \& social isolation that would ultimately leave people more vulnerable to adverse mental health effects caused by the pandemic [15, 16, 17, 18]. The findings in our study provide one of the earliest evidences to corroborate this concern by highlighting the unintended consequences that the social distancing mantra had caused to individuals' social support activation mechanism.

Social settings like shopping venues, schools, bars, restaurants, places of worship, while serving as contexts for physical transmission of the SARS-CoV-2 virus, also serve as an important conduit through which they could activate \& receive social support from their strong ties whenever they faced distress. This latter role, which would be essential in mitigating the mental health impacts of the COVID-19 pandemic, appears to have been recognised much less in the drafting of current pandemic control measures. By simply restricting people's access to these contexts without providing alternatives, current social distancing measures had inadvertently acted as a policy that restricted people's means of help-seeking. In doing so, these measures had effectively increased the bar for people to activate their strong ties as a response against distress, leaving them with no protective buffer until later stages of mental health problems.

Simultaneously, the current findings also call for renewed attention towards the drive in advancing youths' mental health. Prior to the pandemic, given youths' low predisposition to seek help for their mental health problems [42, 43, 44], stakeholders had been pushing for individuals to seek help as early as possible through means such as lowering stigma against help-seeking for mental health problems [45], mental health literacy campaigns [46], and encouraging increased usage of mental health services [47]. Given that strong ties are predominantly the first safety buffer for individuals against mental health impacts of adverse life events [42], the increased threshold for youths to start seeking help from these ties thus serves as an early warning signal for stakeholders such that timely action can be taken to prevent the progress achieved in recent years from being further stalled or worse, undone.

Policies and population health messaging are powerful tools in mitigating mental health impacts of the COVID-19 pandemic [48], but as evidenced in this study, the current emphasis on "social distancing" needs to be reversed. Moving forward, authorities and policymakers should revise the essence of their messaging to "physically distant yet socially close" in order to (i) again remind youths in the community to reach out for help early when they face mental health problems; and also (ii) spur the community to reconfigure / adjust their mechanisms of social support activation in the pandemic era.

Empowering alternative social support channels. Technological advancements have seen new conduits for providing social support, e.g., Internet-delivered counseling, and fostering new bonding social capital, e.g., online peer communities [49] that have remained intact during the pandemic. More resources can therefore be devoted in awareness campaigns for these online platforms and in facilitating existing offline services to extend their services online. Moreover, investing these resources now would not only be cost-effective in improving youths' mental health in the short run (as youths were more receptive than previous generations to these newer support channels [50]), but as more and more of our daily lives are integrated online, would also be good for population mental health in the long run.

Mental health problem resolution starts from the family. Family members and friends are often the first points of contact whom individuals with mental health conditions reach out to [42]. Given that a majority of youths studied here lived with family members, as shown in Supplementary Table S3, it is thus concerning that they sought help less from those who lived in close proximity to them during the pandemic. Most commonly reported setbacks in seeking help from strong ties include inappropriate support, stigma, and the supporter's lack of training and knowledge [51]. Hence, especially during this pandemic, more awareness campaigns to encourage people to seek help early should be held. Furthermore, there should also be stepped-up efforts in encouraging the community to actively maintain social connectedness by checking in on their family \& friends, as well as in educating the community to spot early warning signs of mental health issues in their family members \& immediate circle of friends and to provide effective mental health first-aid support.

There are two main limitations in this study. First, due to the participant recruitment procedures, the study population may not be representative of the general youth population in Hong Kong; individuals with mental health conditions may have been represented more (yet, we argue that these at-risk individuals are precisely the 
ones who need more of our attention). No question on ethnicity was also asked in the survey, and thus we could not ascertain whether ethnic minority youths, who made up around $1 \%$ of the total youths living in Hong Kong according to the latest by-census [38], had been proportionally represented in the study population.

Second, Hong Kong was rocked by social unrest that lasted from mid-2019 to the beginning of 2020, the biggest demographic constituent of which were youths. Cases of breakdowns in family relationships sparked by the unrest had been highlighted on news reports. Since the data collection period for the $2^{\text {nd }}$ wave of the survey only coincided with the first half of the social unrest, we thus could not rule out the possibility that the second half of the unrest had confounded help-seeking preferences from the family in the $3^{\text {rd }}$ wave.

Nevertheless, the findings here aim to provide concrete, evidence-based impetus for stakeholders and authorities around the world that are still using the term "social distancing" to immediately revise their messaging, so as to prevent the current pandemic from turning into a mental health pandemic. Finally, moving forward, the essence of population health messaging in combating the pandemic should be revised to encourage people to be socially close even though they may be physically distant. We call for a concerted effort in empowering alternative social support channels such as e-mental health channels and also the community through awareness \& mental health first-aid training campaigns to further this agenda. 


\section{Methods}

\section{Participants \& procedures}

As part of a broader cross-institutional drive for suicide prevention among youths in Hong Kong, three waves of an online survey targeting youths living in the general Hong Kong community between 11 and 35 years old were conducted annually from 2018 to 2020 . This initiative was led by the Centre for Suicide Research and Prevention (CSRP) at the University of Hong Kong (HKU).

For maximum outreach to the community, each survey wave was disseminated on multiple outlets: As poster promotions at the authors' institution and three large nonprofit organizations in the territory which provide various pastoral services - Caritas, Hong Kong Federation of Youth Groups, and The Boys' and Girls' Clubs Association of Hong Kong; reminders sent to the three organizations' members; bulk emails to newsletter subscribers of CSRP as well as students \& staffs of HKU; and posts on CSRP's Facebook and web page. Moreover, participants from the previous wave who consented to be contacted further were also reached.

Participants could choose to fill either a Chinese or English version of the survey. Written informed consent was first obtained from all participants, and they were informed of the survey's purpose (gaining an in-depth understanding of youths' general wellbeing), approximate survey duration (ten minutes), strict confidentiality of their data, and of their freedom to discontinue at any time. Careful consideration was taken to ensure that the survey questions would incur no risk and pose the least stress to participants. Contact information of emotional support hotlines and services were made available in the survey to encourage any distressed participant to seek support immediately.

All procedures \& protocols adopted in this study complied with ethical standards stipulated in the Helsinki Declaration and were approved by the Human Research Ethics Committee for Non-Clinical Faculties of HKU under the reference number EA1709039. Consent from parents or legal guardians for under-aged participants was deemed not required by the committee as the endorsed study was assessed to pose minimal potential harm to under-aged participants.

The $2^{\text {nd }}$ and $3^{\text {rd }}$ waves were conducted from 5 June 2019 to 8 July 2019 and from 29 June 2020 to 29 September 2020 respectively. The novel SARS-CoV-2 virus was confirmed to have spread to Hong Kong on 22 January 2020, and subsequently the government introduced social distancing measures that were strongly enforced from 29 January 2020, which involved, among others, restricting dining and closing down bars, schools, recreational spaces, and places of worship. Thus, the $2^{\text {nd }}$ and $3^{\text {rd }}$ waves of the survey provide measures on these individuals before and during the COVID-19 pandemic respectively, or alternatively, under the absence and presence of social distancing measures. We only included individuals who completed both waves of the survey in our analysis.

\section{Measures}

\section{Demographics}

Participants were first surveyed on their gender, age, education level, occupation, family structure, and members they currently lived with.

\section{Stressors and psychological distress}

The questionnaire then asked for participants to rate how much distress they experienced in 8 aspects of their lives within the past 4 weeks: academic, job, financial, social life (pertaining to colleagues, friends, classmates), physical well-being, emotional well-being, relations with family, and relations with partner or spouse - from 1 ("not at all") to 5 ("very serious"), or "not applicable". For each participant, we then excluded items with "not applicable" responses and obtained an average of the remaining self-rated stress levels as a proxy for the participant's mental health.

Similarly, the questionnaire also measured participants' psychological distress within the past 1 to 2 weeks with the 12-Item Chinese Health Questionnaire (CHQ-12), where each item was scored on a 4-point scale - from 1 ("not at all") to 4 ("much more than usual") - and a higher total score indicating heavier psychological distress $[52,53,54]$. 


\section{Risk behaviours}

Participants were then prompted whether they had considered suicide, attempted suicide, or performed intentional self-harm in the past 12 months. All three questions were binary ("yes" and "no") questions.

Social engagement of participants was measured with the Social Engagement-Hikikomori Scale [55] to screen for social withdrawal. A participant who responded "yes" to "spending most of the day and nearly every day confined at home" \& "persistently avoiding social situations and social contact" and had not been diagnosed with any listed psychiatric disorder would be deemed as a Hikikomori, i.e., a socially withdrawn person [56].

\section{Help-seeking behaviours}

Lastly, participants were asked on which of 10 support channels had they activated to deal with the above stressors within the past 4 weeks: (1) family members; (2) friends, classmates, or colleagues; (3) spouse or partner; (4) teachers or tutors; (5) free hotline support; (6) medical professionals; (7) social workers or counsellors; (8) religious services; (9) online friends whom they had never met physically; and (10) online social services. The questions were also binary questions and participants could choose all that applied to them.

\section{Statistical analyses}

We first measured descriptive statistics of the study population. We then tested for H1 by employing Welch's $t$-test and Fisher's exact test to examine trends in help-seeking tendencies of the study population between the two survey waves. Effect sizes of between-wave differences were quantified by Cohen's $d$ and odds ratios. Trends in stress levels and risk behaviours were also examined with the same approach. All analyses were conducted on $\mathrm{R}$, and throughout our analyses, a $p$-value of less than 0.05 was considered to be statistically significant.

We then conducted a latent class analysis (LCA) of the 10 support channels because doing so allows us to disentangle individuals' help-seeking behaviours from distress \& sociodemographic factors, and thereby classify individuals based on their help-seeking patterns. This was implemented using the poLCA package [57] in R version 4.0.3. A separate LCA was conducted for each wave in order to identify individuals whose help-seeking patterns changed between the two survey waves.

For each LCA, we started from a model with 1 latent class and sequentially increased the number of classes up to 5 to find the optimum number. At each subsequent step, we evaluated the model based on 4 model-fit parameters that had been established as the most reliable for LCA with categorical outcomes - the bootstrap likelihood ratio test (BLR) [58], Bayesian information criterion (BIC) [59], sample size-adjusted BIC (SABIC) [60], and the Lo-Mendell-Rubin (LMR) likelihood ratio test [61] - as well as the interpretability of each class $[62,63,64]$. We also made sure that each model iteration had converged before being evaluated. The model yielding approximately the lowest BIC \& SABIC, significant $p$-values for the BLR \& LMR tests, and an intuitive interpretation of help-seeking behaviours for each of its latent class was chosen (see Model selection). The number of latent classes in this model then corresponded to the number of unique help-seeking patterns among the analyzed study population.

Finally, we conducted multiple logistic regression with between-wave stress level difference \& sociodemographic factors as predictors and between-wave shift in help-seeking pattern as the outcome variable. This is to ascertain whether individuals whose shifts in help-seeking patterns showed reduced help-seeking in 2020 were associated with specific subgroups that were thought to be less affected by the pandemic. In our case, statistical insignificance for all predictors would support the hypothesis that a lower inclination for these individuals to seek help in response to distress was indeed a consequence of social distancing measures. 


\section{References}

[1] Ichiro Kawachi and Lisa F Berkman. Social ties and mental health. Journal of Urban Health, 78(3):458-467, 2001.

[2] Nan Lin. Social capital: A theory of social structure and action. Cambridge University Press, 2002.

[3] John Taylor and R Jay Turner. A longitudinal study of the role and significance of mattering to others for depressive symptoms. Journal of Health and Social Behavior, pages 310-325, 2001.

[4] Peggy A Thoits. Mechanisms linking social ties and support to physical and mental health. Journal of health and social behavior, 52(2):145-161, 2011.

[5] Ziggi Ivan Santini, Ai Koyanagi, Stefanos Tyrovolas, Catherine Mason, and Josep Maria Haro. The association between social relationships and depression: a systematic review. Journal of affective disorders, 175:53-65, 2015.

[6] Brea L Perry and Bernice A Pescosolido. Social network activation: the role of health discussion partners in recovery from mental illness. Social science $\mathcal{E}$ medicine, 125:116-128, 2015.

[7] Spencer Moore and Richard M Carpiano. Introduction to the special issue on "social capital and health: What have we learned in the last 20 years and where do we go from here?", 2020.

[8] Robert D Putnam et al. Bowling alone: The collapse and revival of American community. Simon and Schuster, 2000.

[9] Wouter Poortinga. Social relations or social capital? Individual and community health effects of bonding social capital. Social science 83 medicine, 63(1):255-270, 2006.

[10] Daniel Kim, Sukanya V Subramanian, and Ichiro Kawachi. Bonding versus bridging social capital and their associations with self rated health: a multilevel analysis of 40 US communities. Journal of Epidemiology $\mathcal{G}$ Community Health, 60(2):116-122, 2006.

[11] Jay Irwin, Mark LaGory, Ferris Ritchey, and Kevin Fitzpatrick. Social assets and mental distress among the homeless: Exploring the roles of social support and other forms of social capital on depression. Social Science \&3 Medicine, 67(12):1935-1943, 2008.

[12] Lijun Song. Social capital and psychological distress. Journal of health and social behavior, 52(4):478-492, 2011.

[13] Eran P Melkman. Childhood adversity, social support networks and well-being among youth aging out of care: An exploratory study of mediation. Child abuse $\mathcal{E}$ neglect, 72:85-97, 2017.

[14] Mattia Vacchiano and Danilo Bolano. Online and offline leisure, relatedness and psychological distress: a study of young people in Switzerland. Leisure Studies, pages 1-14, 2020.

[15] Danuta Wasserman, Rutger van der Gaag, and Jan Wise. The term "physical distancing" is recommended rather than "social distancing" during the covid-19 pandemic for reducing feelings of rejection among people with mental health problems. European Psychiatry, 63(1), 2020.

[16] Reza Aminnejad and Rosa Alikhani. Physical distancing or social distancing: that is the question. Canadian Journal of Anesthesia/Journal canadien d'anesthésie, 67:1457-1458, 2020.

[17] Thomas Abel and David McQueen. The covid-19 pandemic calls for spatial distancing and social closeness: not for social distancing!, 2020.

[18] Christine Moutier. Suicide prevention in the covid-19 era: transforming threat into opportunity. JAMA psychiatry, 78(4):433-438, 2021.

[19] Sandro Galea, Raina M Merchant, and Nicole Lurie. The mental health consequences of covid-19 and physical distancing: the need for prevention and early intervention. JAMA internal medicine, 180(6):817-818, 2020.

[20] Christoph Benke, Lara K Autenrieth, Eva Asselmann, and Christiane A Pané-Farré. Lockdown, quarantine measures, and social distancing: Associations with depression, anxiety and distress at the beginning of the covid-19 pandemic among adults from germany. Psychiatry research, 293:113462, 2020. 
[21] Ingibjorg Eva Thorisdottir, Bryndis Bjork Asgeirsdottir, Alfgeir Logi Kristjansson, Heiddis Bjork Valdimarsdottir, Erla Maria Jonsdottir Tolgyes, Jon Sigfusson, John Philip Allegrante, Inga Dora Sigfusdottir, and Thorhildur Halldorsdottir. Depressive symptoms, mental wellbeing, and substance use among adolescents before and during the covid-19 pandemic in iceland: a longitudinal, population-based study. The Lancet Psychiatry, 2021.

[22] Debra J Rickwood and Valerie A Braithwaite. Social-psychological factors affecting help-seeking for emotional problems. Social Science \& Medicine, 39(4):563-572, 1994.

[23] Ness T Morgan and M Robinson. Students' help-seeking behaviours by gender, racial background, and student status. Canadian Journal of Counselling, 37(2):151-166, 2003.

[24] Matthew Hotopf, Ed Bullmore, Rory C O'Connor, and Emily A Holmes. The scope of mental health research during the COVID-19 pandemic and its aftermath. The British Journal of Psychiatry, 217(4):540-542, 2020.

[25] Emily A Holmes, Rory C O'Connor, V Hugh Perry, Irene Tracey, Simon Wessely, Louise Arseneault, Clive Ballard, Helen Christensen, Roxane Cohen Silver, Ian Everall, et al. Multidisciplinary research priorities for the covid-19 pandemic: a call for action for mental health science. The Lancet Psychiatry, 2020.

[26] Jörg M Fegert, Benedetto Vitiello, Paul L Plener, and Vera Clemens. Challenges and burden of the Coronavirus 2019 (COVID-19) pandemic for child and adolescent mental health: a narrative review to highlight clinical and research needs in the acute phase and the long return to normality. Child and adolescent psychiatry and mental health, 14:1-11, 2020.

[27] Dirk Richter, Steffi Riedel-Heller, and Simeon Joel Zürcher. Mental health problems in the general population during and after the first lockdown phase due to the SARS-Cov-2 pandemic: rapid review of multi-wave studies. Epidemiology and Psychiatric Sciences, 30:e27, 2021.

[28] Tilmann von Soest, Michal Kozák, Ruben Rodriguez-Cano, Dirkje Fluit, Laura Cortés-García, Vidar Ulseth, EF Haghish, and Anders Bakken. A nationwide study of adolescent psychosocial well-being one year after the outbreak of the covid-19 pandemic. under review, 2021.

[29] Mark Shevlin, Orla McBride, Jamie Murphy, Jilly Gibson Miller, Todd K Hartman, Liat Levita, Liam Mason, Anton P Martinez, Ryan McKay, Thomas VA Stocks, et al. Anxiety, depression, traumatic stress and COVID-19-related anxiety in the UK general population during the COVID-19 pandemic. BJPsych Open, 6(6), 2020.

[30] Matthias Pierce, Holly Hope, Tamsin Ford, Stephani Hatch, Matthew Hotopf, Ann John, Evangelos Kontopantelis, Roger Webb, Simon Wessely, Sally McManus, et al. Mental health before and during the COVID19 pandemic: a longitudinal probability sample survey of the UK population. The Lancet Psychiatry, $7(10): 883-892,2020$.

[31] Nina Vindegaard and Michael Eriksen Benros. Covid-19 pandemic and mental health consequences: Systematic review of the current evidence. Brain, behavior, and immunity, 89:531-542, 2020.

[32] Eleonora Iob, Philipp Frank, Andrew Steptoe, and Daisy Fancourt. Levels of severity of depressive symptoms among at-risk groups in the UK during the COVID-19 pandemic. JAMA network open, 3(10):e2026064e2026064, 2020.

[33] Eleonora Iob, Andrew Steptoe, and Daisy Fancourt. Abuse, self-harm and suicidal ideation in the uk during the covid-19 pandemic. The British Journal of Psychiatry, 217(4):543-546, 2020.

[34] Qingqing Xu, Zhenxing Mao, Dandan Wei, Pengling Liu, Keliang Fan, Juan Wang, Xian Wang, Xiaomin Lou, Hualiang Lin, Chongjian Wang, et al. Prevalence and risk factors for anxiety symptoms during the outbreak of COVID-19: A large survey among 373216 junior and senior high school students in China. Journal of Affective Disorders, 288:17-22, 2021.

[35] Jack Tsai, Eric B Elbogen, Minda Huang, Carol S North, and Robert H Pietrzak. Psychological distress and alcohol use disorder during the COVID-19 era among middle-and low-income US adults. Journal of affective disorders, 288:41-49, 2021.

[36] Cuiyan Wang, Riyu Pan, Xiaoyang Wan, Yilin Tan, Linkang Xu, Roger S McIntyre, Faith N Choo, Bach Tran, Roger Ho, Vijay K Sharma, et al. A longitudinal study on the mental health of general population during the COVID-19 epidemic in China. Brain, behavior, and immunity, 87:40-48, 2020. 
[37] Cedric Sachser, Gabriel Olaru, Elisa Pfeiffer, Elmar Brähler, Vera Clemens, Miriam Rassenhofer, Andreas Witt, and Jörg M Fegert. The immediate impact of lockdown measures on mental health and couples' relationships during the COVID-19 pandemic - Results of a representative population survey in Germany. Social Science 83 Medicine, page 113954, 2021.

[38] Census and HKSAR Government Statistics Department. Main Tables - 2016 Population By-census. https: //www. bycensus2016.gov.hk/en/bc-mt.html, 2017.

[39] Fiona Judd, Angela Komiti, and Henry Jackson. How does being female assist help-seeking for mental health problems? Australian 83 New Zealand Journal of Psychiatry, 42(1):24-29, 2008.

[40] Sarah Clement, Oliver Schauman, Tanya Graham, F Maggioni, Sara Evans-Lacko, Nikita Bezborodovs, Craig Morgan, Nicolas Rüsch, June SL Brown, and Graham Thornicroft. What is the impact of mental healthrelated stigma on help-seeking? A systematic review of quantitative and qualitative studies. Psychological Medicine, 45(1):11-27, 2015.

[41] Debra Rickwood, Frank P Deane, Coralie J Wilson, and Joseph Ciarrochi. Young people's help-seeking for mental health problems. Australian e-journal for the Advancement of Mental Health, 4(3):218-251, 2005.

[42] Debra J Rickwood, Frank P Deane, and Coralie J Wilson. When and how do young people seek professional help for mental health problems? Medical journal of Australia, 187(S7):S35-S39, 2007.

[43] Phoenix KH Mo and Winnie WS Mak. Help-seeking for mental health problems among Chinese. Social Psychiatry and Psychiatric Epidemiology, 44(8):675-684, 2009.

[44] Louisa Picco, Edimansyah Abdin, Siow Ann Chong, Shirlene Pang, Janhavi A Vaingankar, Vathsala Sagayadevan, Kian Woon Kwok, and Mythily Subramaniam. Beliefs about help seeking for mental disorders: Findings from a mental health literacy study in singapore. Psychiatric Services, 67(11):1246-1253, 2016.

[45] Daniel Eisenberg, Marilyn F Downs, Ezra Golberstein, and Kara Zivin. Stigma and help seeking for mental health among college students. Medical Care Research and Review, 66(5):522-541, 2009.

[46] Claire M Kelly, Anthony F Jorm, and Annemarie Wright. Improving mental health literacy as a strategy to facilitate early intervention for mental disorders. Medical Journal of Australia, 187(S7):S26-S30, 2007.

[47] Mark Olfson, Benjamin G Druss, and Steven C Marcus. Trends in mental health care among children and adolescents. New England Journal of Medicine, 372(21):2029-2038, 2015.

[48] Jay J Van Bavel, Katherine Baicker, Paulo S Boggio, Valerio Capraro, Aleksandra Cichocka, Mina Cikara, Molly J Crockett, Alia J Crum, Karen M Douglas, James N Druckman, et al. Using social and behavioural science to support covid-19 pandemic response. Nature human behaviour, 4(5):460-471, 2020.

[49] Munmun De Choudhury and Sushovan De. Mental health discourse on reddit: Self-disclosure, social support, and anonymity. In Proceedings of the International AAAI Conference on Web and Social Media, volume 8, 2014.

[50] Tracey A Davenport, Vanessa Wan Sze Cheng, Frank Iorfino, Blake Hamilton, Eva Castaldi, Amy Burton, Elizabeth M Scott, and Ian B Hickie. Flip the clinic: A digital health approach to youth mental health service delivery during the covid-19 pandemic and beyond. JMIR mental health, 7(12):e24578, 2020.

[51] Kathleen M Griffiths, Dimity A Crisp, Lisa Barney, and Russell Reid. Seeking help for depression from family and friends: a qualitative analysis of perceived advantages and disadvantages. BMC psychiatry, 11(1):1-12, 2011.

[52] Tiffany Szu-Ting Fu, Chau-Shoun Lee, David Gunnell, Wen-Chung Lee, and Andrew Tai-Ann Cheng. Changing trends in the prevalence of common mental disorders in Taiwan: a 20-year repeated cross-sectional survey. The Lancet, 381(9862):235-241, 2013.

[53] Tai-Ann Cheng and Paul Williams. The design and development of a screening questionnaire (CHQ) for use in community studies of mental disorders in Taiwan. Psychological medicine, 16(2):415-422, 1986.

[54] Pey-Chyou Pan and David P Goldberg. A comparison of the validity of GHQ-12 and CHQ-12 in Chinese primary care patients in Manchester. Psychological Medicine, 20(4):931-940, 1990. 
[55] Alan Robert Teo and Albert C Gaw. Hikikomori, a Japanese culture-bound syndrome of social withdrawal? A proposal for DSM-V. The Journal of nervous and mental disease, 198(6):444, 2010.

[56] Paul WC Wong, Tim MH Li, Melissa Chan, YW Law, Michael Chau, Cecilia Cheng, KW Fu, John BaconShone, and Paul SF Yip. The prevalence and correlates of severe social withdrawal (hikikomori) in Hong Kong: A cross-sectional telephone-based survey study. International Journal of Social Psychiatry, 61(4):330$342,2015$.

[57] Drew Linzer and Jeffrey Lewis. poLCA: An R package for polytomous variable latent class analysis. Journal of Statistical Software, Articles, 42(10):1-29, 2011.

[58] Geoffrey J McLachlan. On bootstrapping the likelihood ratio test statistic for the number of components in a normal mixture. Journal of the Royal Statistical Society: Series C (Applied Statistics), 36(3):318-324, 1987.

[59] Gideon Schwarz et al. Estimating the dimension of a model. The Annals of Statistics, 6(2):461-464, 1978.

[60] Stanley L Sclove. Application of model-selection criteria to some problems in multivariate analysis. Psychometrika, 52(3):333-343, 1987.

[61] Yungtai Lo, Nancy R Mendell, and Donald B Rubin. Testing the number of components in a normal mixture. Biometrika, 88(3):767-778, 2001.

[62] Karen L Nylund, Tihomir Asparouhov, and Bengt O Muthén. Deciding on the number of classes in latent class analysis and growth mixture modeling: A Monte Carlo simulation study. Structural Equation Modeling: A multidisciplinary Journal, 14(4):535-569, 2007.

[63] Karen Nylund, Amy Bellmore, Adrienne Nishina, and Sandra Graham. Subtypes, severity, and structural stability of peer victimization: What does latent class analysis say? Child Development, 78(6):1706-1722, 2007.

[64] Linda M Collins and Stephanie T Lanza. Latent class and latent transition analysis: With applications in the social, behavioral, and health sciences, volume 718. John Wiley \& Sons, 2009.

\section{Declarations}

\section{Conflicts of Interest: None.}

Funding Statement: This work was supported by the Hong Kong Charities Trust and the Humanities and Social Science Prestigious Fellowship [PY].

Acknowledgements: We would like to thank the three non-profit organizations (Caritas, The Hong Kong Federation of Youth Groups, The Boys' and Girls' Clubs Association of Hong Kong) and members of the Evaluation and Knowledge Dissemination Subcommittee of the HKJC Online Youth Emotional Support project, who helped disseminate and collect the survey data.

Author Contributions: AJ \& CK conceived the presented idea, set the proof outline, and wrote the manuscript with input from all authors. AJ, CK, CW performed the computations and verified the analytical methods. ZC \& PY reviewed and supervised the findings of this work. All authors discussed the results and contributed to the final manuscript.

Data availability: The data that support the findings of this study are available from the corresponding author upon reasonable request. 


\section{Supplementary Files}

This is a list of supplementary files associated with this preprint. Click to download.

- SuppInfoSciRep.pdf 